Annals of Pure and Applied Mathematics

Vol. 21, No. 1, 2020, 19-25

ISSN: 2279-087X (P), 2279-0888(online)

Published on 16 February 2020

www.researchmathsci.org

DOI: http://dx.doi.org/10.22457/apam.v21n1a3649

Annals of

Pure and Applied Mathematics

\title{
Some Properties of Double Minimal Space
}

\author{
Dheargham Ali Abdulsada \\ Department of Statistics, Faculty of Administration and Economics \\ Sumer University, Iraq. Email: drghamali1985@gmail.com
}

Received 28 December 2019; accepted 29 January 2020

\begin{abstract}
In this paper, we present some important concepts indouble minimal space and study of (interior, closure) sets this space. Moreover, we give concepts Regular $\mathfrak{M}_{\mathrm{D}}$-(open, closed) sets, a $\mathfrak{M}_{\mathrm{D}}-(\mathcal{A}, \mathrm{t}, \mathfrak{B}, \mathfrak{h}$ and $\mathcal{C})$ sets and find the relationship between these concepts.
\end{abstract}

Keywords: Double set; Double minimal structure; Regular $-\mathfrak{M}_{\mathrm{D}}-$ (open, closed) sets; a $\mathfrak{M}_{\mathrm{D}^{-}}(\mathcal{A}, \mathrm{t}, \mathfrak{B}, \mathfrak{h}$ and $\mathcal{C})$ sets.

AMS Mathematics Subject Classification (2010): 53A07

\section{Introduction}

Fuzzy sets presented by Zadeh [7] in 1965 showed many important applications in many areas of life. The fuzzy set was used to tackle problems characterized by ambiguity and immunity that classic sets were unable to address.Fuzzy sets. The possibility of the fuzzy set has been invited as it handles vulnerability and ambiguity which Cantorian set couldn't address. Speculation of fuzzy sets was in this manner proposed by Atanassov [1] 1983 as intuitionistic fuzzy sets (IFS) which consolidate the level of dithering called faltering edge.The information and semantic portrayal of intuitionistic fuzzy set has gotten progressively significant, clever and material, since it incorporates the level of belongings, the level of non-belongings and the faltering edge. Then again, Coker [3] presented the discrete type of intuitionistic fuzzy set to be specific 'intuitionistic sets in 1996, where every one of the sets is completely fresh sets. In any case, it has an enrollment and 3 non-participation degrees and thus this idea gives us increasingly adaptable methodologies in speaking to unclearness in scientific articles incorporating those in building fields with traditional set rationale. In the year 2000, Coker [4] additionally presented the idea of intuitionistic topological spaces with intuitionistic set and explored their properties. In 2012, Adak, Bhowmik and Pal, [2] introduce Intuitionistic fuzzy block matrix and its some properties. Bhowmik and Pal defined intuitionistic fuzzy circulant matrix [9]. In 2015, Ahmed, Hossain and Ali [5] they did a study intuitionistic fuzzy R0-spaces. In 2017, Islam, Hossain and Amin [8] they studied a conceptSome Remarks on Intuitionistic L- $T_{2}$ Spaces. In this paper, we pursue the proposal of Garcia and Rodabaugh [6] that "double fuzzy set" is a more proper name than "intuitionistic fuzzy set", and, subsequently, receive the expression "double set" for the intuitionistic set. We define minimal double space and study its properties. In addition to defining the concepts Regular $-\mathfrak{M}_{\mathrm{D}}-$ (open, closed) sets, a $\mathfrak{M}_{\mathrm{D}}-(\mathcal{A}, \mathrm{t}, \mathfrak{B}, \mathfrak{h}$ and $\mathcal{C})$ 


\section{Dheargham Ali Abdulsada}

sets and find the relationship between these concepts. Bhowmik and Pal [10] defined generalized intuitionstic fuzzy sets.

\section{Preliminaries}

In this section, we remember some basic concepts related to double groups.

Definition 2.1. [3] A double set (DS for short) $\ddot{A}$ of a non-void set $\mathrm{X}$ is $\ddot{A}=\left\langle A^{\prime}, A^{\prime \prime}\right\rangle$ where $A^{\prime}, A^{\prime \prime} \subseteq \mathrm{X}$ and $A^{\prime} \cap A^{\prime \prime}=\varnothing$.

Definition 2.2.[3] If $\ddot{A}=\left\langle A^{\prime}, A^{\prime \prime}\right\rangle, \ddot{B}=\left\langle B^{\prime}, B^{\prime \prime}\right\rangle$ and $\left\{\ddot{A}_{i}: i \in I\right\}$ are double sets in $X$, where $\ddot{A}_{i}=\left\langle A^{\prime}{ }_{i}, A^{\prime \prime}{ }_{i}\right\rangle$. Then

1. $\ddot{A} \subseteq \subseteq_{D} \ddot{B} \Leftrightarrow A^{\prime} \subseteq B^{\prime} \& B^{\prime \prime} \subseteq A^{\prime \prime}$.

2. $\ddot{A}=\ddot{B} \Leftrightarrow \ddot{A} \subseteq_{D} \ddot{B} \& \ddot{B} \subseteq_{D} \ddot{A}$.

3. $\cup_{D} \ddot{A}_{i}=\left\langle\cup A^{\prime}{ }_{i}, \cap A^{\prime \prime}{ }_{i}\right\rangle$.

4. $\cap_{D} \ddot{A}_{i}=\left\langle\cap A^{\prime}{ }_{i}, \cup A^{\prime \prime}{ }_{i}\right\rangle$.

5. $\ddot{\mathrm{X}}_{D}=\langle\mathrm{X}, \emptyset\rangle$.

6. $\ddot{\emptyset}_{D}=\langle\emptyset, X\rangle$.

7. $\operatorname{cop} . \ddot{A}=\left\langle A^{\prime \prime}, A^{\prime}\right\rangle$.

8. $\ddot{A} \backslash \ddot{B}=\ddot{A} \cap_{D} \ddot{B}$.

Definition 2.3.[3] A double point of a non-void set $X$ is $\ddot{p}_{D}=\left\langle\{p\},\{p\}^{c}\right\rangle$, where $p \in X$.

\section{Double minimal structure}

In this section, we will study the concept of minimal structure by double set and explain some of its characteristics.

Definition 3.1. A double minimal structure $\left(\mathfrak{M}_{\mathrm{D}}\right.$-structure for $)$ on a non-void set $\mathrm{X}$ is a collection $\mathfrak{M}_{\mathrm{DX}}$ of double sets in X, where $\ddot{X}_{D} \in \mathfrak{M}_{\mathrm{DX}}$ and $\ddot{\emptyset}_{D} \in \mathfrak{M}_{\mathrm{DX}}$.

A set $X$ with $\mathfrak{M}_{X}$ is called an $\mathfrak{M}_{\mathrm{DX}^{-}}$-space and is denoted by $\left(\mathrm{X}, \mathfrak{M}_{\mathrm{DX}}\right)$. Each member of $\mathfrak{M}_{\mathrm{DX}}$ is said to be $\mathfrak{M}_{\mathrm{D}^{-}}$-open and the double complement of an $\mathfrak{M}_{\mathrm{D}^{-}}$-open set is said to be $\mathfrak{M}_{\mathrm{DX}}$-closed.

Definition 3.2. Let $X$ be a nonempty set and $\mathfrak{M}_{\mathrm{DX}}$ an $\mathfrak{M}_{\mathrm{D}}$-structure on $\mathrm{X}$. For a double set $\ddot{A}$ of $\mathrm{X}$, the $\mathfrak{M}_{\mathrm{DX}}$-closure of $\ddot{A}$ and the $\mathfrak{M}_{\mathrm{DX}}$-interior of $\ddot{A}$. The double minimal structureis defined as follows:

1. $\mathfrak{M}_{\mathrm{DX}}-\operatorname{Cl}(\ddot{A})=\cap_{D}\left\{\ddot{F}=\left\langle F^{\prime}, F^{\prime \prime}\right\rangle: \ddot{A} \subseteq \subseteq_{D} \ddot{F}, \ddot{F}\right.$ is $\mathfrak{M}_{\mathrm{D}}-$ closed $\}$,

2. $\mathfrak{M}_{\mathrm{DX}}-\operatorname{int}(\ddot{A})=\cup_{D}\left\{\ddot{U}=\left\langle U^{\prime}, U^{\prime \prime}\right\rangle: \ddot{U} \subseteq_{D} \ddot{A}, \ddot{U}\right.$ is $\mathfrak{M}_{\mathrm{D}}-$ open $\}$

Lemma 3.3. Let $X$ be a nonempty set and $\mathfrak{M}_{\mathrm{DX}}$ a on $X$. For double sets $\ddot{A}$ and $\ddot{B}$ of $X$, the following properties hold :

1. $\mathfrak{M}_{\mathrm{DX}}-\operatorname{Cl}(\operatorname{cop} . \ddot{A})=\operatorname{cop} . \mathfrak{M}_{\mathrm{DX}}-\operatorname{int}(\ddot{A})$ and $\mathfrak{M}_{\mathrm{DX}}-\operatorname{int}(\operatorname{cop} . \ddot{A})=\operatorname{cop} . \mathfrak{M}_{\mathrm{DX}}-$ $\operatorname{cl}(\ddot{A})$, 
Some Properties of Double Minimal Space

2. If cop. $\ddot{A} \in \mathfrak{M}_{\mathrm{DX}}$, then $\mathfrak{M}_{\mathrm{DX}}-\operatorname{cl}(\ddot{A})=\ddot{A}$ and if $\ddot{A} \in \mathfrak{M}_{\mathrm{DX}}$, then $\mathfrak{M}_{\mathrm{DX}}-\operatorname{int}(\ddot{A})=$ $\ddot{A}$,

3. $\mathfrak{M}_{\mathrm{DX}}-\operatorname{int}\left(\ddot{\emptyset}_{D}\right)=\ddot{\emptyset}_{D}, \quad \mathfrak{M}_{\mathrm{DX}}-\operatorname{int}\left(\ddot{\mathrm{X}}_{D}\right)=\ddot{\mathrm{X}}_{D}, \quad \mathfrak{M}_{\mathrm{DX}}-\operatorname{cl}\left(\ddot{\emptyset}_{D}\right)=\ddot{\emptyset}_{D}, \quad \mathfrak{M}_{\mathrm{DX}}-$ $\operatorname{cl}\left(\ddot{\mathrm{X}}_{D}\right)=\ddot{\mathrm{X}}_{D}$

4. If $\ddot{A} \subseteq_{D} \ddot{B}$, then $\mathfrak{M}_{\mathrm{DX}}-\operatorname{int}(\ddot{A}) \subseteq_{D} \mathfrak{M}_{\mathrm{DX}}-\operatorname{int}(\ddot{B})$ and $\mathfrak{M}_{\mathrm{DX}}-\operatorname{cl}(\ddot{A}) \subseteq_{D} \mathfrak{M}_{\mathrm{DX}}-$ $\operatorname{cl}(\ddot{B})$,

5. $\ddot{A} \subseteq_{D} \mathfrak{M}_{\mathrm{DX}}-\operatorname{cl}(\ddot{A})$ and $\mathfrak{M}_{\mathrm{DX}}-\operatorname{int}(\ddot{A}) \subseteq_{D} \ddot{A}$.

6. $\mathfrak{M}_{\mathrm{DX}}-\operatorname{cl}\left(\mathfrak{M}_{\mathrm{DX}}-\operatorname{cl}(\ddot{A})\right)=\mathfrak{M}_{\mathrm{DX}}-\operatorname{cl}(\ddot{A})$ and $\mathfrak{M}_{\mathrm{DX}}-\operatorname{int}\left(\mathfrak{M}_{\mathrm{DX}}-\operatorname{int}(\ddot{A})\right)=$ $\mathfrak{M}_{\mathrm{DX}}-\operatorname{int}(\ddot{A})$.

Definition 3.4. A double minimal structure $\mathfrak{M}_{\mathrm{DX}}$ on a nonempty set $X$ is said to have property $B_{D}$ if the double union of any family of subsets belonging to $\mathfrak{M}_{\mathrm{DX}}$ belongs to $\mathfrak{M}_{\mathrm{DX}}$.

Lemma 3.5. The following are equivalent for the double minimal space $\left(X, \mathfrak{M}_{\mathrm{DX}}\right)$.

1. $\mathfrak{M}_{\mathrm{DX}}$ have property $B_{D}$.

2. If $\mathfrak{M}_{\mathrm{DX}}-\operatorname{int}(\ddot{E})=\ddot{E}$, then $\ddot{E} \in \mathfrak{M}_{\mathrm{DX}}$.

3. If $\mathfrak{M}_{\mathrm{DX}}-\operatorname{int}(\ddot{F})=\ddot{F}$, then $\ddot{F} \in \mathfrak{M}_{\mathrm{DX}}$.

Definition 3.6. A double minimal structure $\mathfrak{M}_{\mathrm{DX}}$ on a nonempty set $\mathrm{X}$ is said to have property $I_{D}$ if the finite double intersection of $\mathfrak{M}_{\mathrm{D}}$-open is $\mathfrak{M}_{\mathrm{D}}$-open.

Remark 3.7. For subsets $\ddot{A}$ and $\ddot{B}$ of a double minimal space $\left(\mathrm{X}, \mathfrak{M}_{\mathrm{DX}}\right)$. Satisfying property $I_{D}$, the following holds: $\mathfrak{M}_{\mathrm{DX}}-\operatorname{int}\left(\ddot{A} \cap_{D} \ddot{B}\right)=\mathfrak{M}_{\mathrm{DX}}-\operatorname{int}(\ddot{A}) \cap_{D} \mathfrak{M}_{\mathrm{DX}}-$ $\operatorname{int}(\ddot{B})$.

Example 3.8. For subsets $\ddot{A}$ and $\ddot{B}$ of a double minimal space $\left(\mathrm{X}, \mathfrak{M}_{\mathrm{DX}}\right)$. Satisfying property $B_{D}$, the following does not hold:

$\mathfrak{M}_{\mathrm{DX}}-\operatorname{int}\left(\ddot{A} \cap_{D} \ddot{B}\right)=\mathfrak{M}_{\mathrm{DX}}-\operatorname{int}(\ddot{A}) \cap_{D} \mathfrak{M}_{\mathrm{DX}}-\operatorname{int}(\ddot{B})$.

Let $X=\left\{z_{1}, z_{2}, z_{3}, z_{4}\right\}$,

$$
\mathfrak{M}_{\mathrm{DX}}=\left\{\ddot{\emptyset}_{D}, \ddot{X}_{D},\left\langle\left\{z_{1}\right\},\left\{z_{2}, z_{3}, z_{4}\right\}\right\rangle,\left\langle\left\{z_{1}, z_{2}\right\},\left\{z_{3}, z_{4}\right\}\right\rangle,\left\langle\left\{z_{1}, z_{3}\right\},\left\{z_{2}, z_{4}\right\}\right\rangle,\right.
$$

$\left.\left\langle\left\{z_{2}, z_{3}\right\},\left\{z_{1}, z_{4}\right\}\right\rangle,\left\langle\left\{z_{1}, z_{2}, z_{3}\right\},\left\{z_{4}\right\}\right\rangle\right\}$.

Let $\ddot{A}=\left\langle\left\{\mathrm{z}_{1}, \mathrm{z}_{2}\right\},\left\{\mathrm{z}_{3}, \mathrm{z}_{4}\right\}\right\rangle$ and $\ddot{B}=\left\langle\left\{\mathrm{z}_{2}, \mathrm{z}_{3}\right\},\left\{\mathrm{z}_{1}, \mathrm{z}_{4}\right\}\right\rangle$.

Then $\ddot{A} \cap_{D} \ddot{B}=\left\langle\left\{\mathfrak{z}_{2}\right\},\left\{\mathfrak{z}_{1}, \mathfrak{z}_{3}, \mathfrak{z}_{4}\right\}\right\rangle$. We have $\mathfrak{M}_{\mathrm{DX}}-\operatorname{int}(\ddot{A})=\left\langle\left\{\mathfrak{z}_{1}, \mathrm{z}_{2}\right\},\left\{\mathfrak{z}_{3}, \mathrm{z}_{4}\right\}\right\rangle$;

$\mathfrak{M}_{\mathrm{DX}}-\operatorname{int}(\ddot{B})=\left\langle\left\{\mathfrak{z}_{2}, \mathrm{z}_{3}\right\},\left\{\mathrm{z}_{1}, \mathrm{z}_{4}\right\}\right\rangle$ and

$\mathfrak{M}_{\mathrm{DX}}-\operatorname{int}(\ddot{A}) \cap_{D} \mathfrak{M}_{\mathrm{DX}}-\operatorname{int}(\ddot{B})=\left\langle\left\{\mathfrak{z}_{2}\right\},\left\{\mathfrak{z}_{1}, \mathrm{z}_{3}, \mathrm{z}_{4}\right\}\right\rangle$.

But $\mathfrak{M}_{\mathrm{DX}}-\operatorname{int}\left(\ddot{A} \cap_{D} \ddot{B}\right)=\ddot{\emptyset}_{D}$.

Hence $\mathfrak{M}_{\mathrm{DX}}-\operatorname{int}\left(\ddot{A} \cap_{D} \ddot{B}\right) \neq \mathfrak{M}_{\mathrm{DX}}-\operatorname{int}(\ddot{A}) \cap_{D} \mathfrak{M}_{\mathrm{DX}}-\operatorname{int}(\ddot{B})$.

4. Regular $-\mathfrak{M}_{\mathrm{D}}-\left(\right.$ open, closed) sets, a $\mathfrak{M}_{\mathrm{D}}-(\mathcal{A}, \mathrm{t}, \mathfrak{B}, \mathfrak{h}$ and $\mathcal{C})$ sets

In this section, we introduce a new type of sets as follows: 
Definition 4.1. A doubleset $\ddot{S}$ of a double minimal space $\left(X, \mathfrak{M}_{\mathrm{DX}}\right)$ is said to be

1. Regular $-\mathfrak{M}_{\mathrm{D}}-$ open if $\ddot{S}=\mathfrak{M}_{\mathrm{D}}-\operatorname{int}\left(\mathfrak{M}_{\mathrm{D}}-\operatorname{cl}(\ddot{S})\right)$,

2. Regular $-\mathfrak{M}_{\mathrm{D}}-$ closed if $\ddot{S}=\mathfrak{M}_{\mathrm{D}}-\operatorname{cl}\left(\mathfrak{M}_{\mathrm{D}}-\operatorname{int}(\ddot{S})\right)$,

The family of all Regular $-\mathfrak{M}_{\mathrm{D}}-$ closed sets of $\mathrm{X}$ is denoted $\mathfrak{M}_{\mathrm{D}}-\mathrm{RC}(\mathrm{X})$.

Definition 4.2. A double set $\ddot{S}$ of a double minimal space $\left(\mathrm{X}, \mathfrak{M}_{\mathrm{DX}}\right)$ is said to be

1. a $\mathfrak{M}_{\mathrm{D}^{-}} \mathcal{A}$ set if $\ddot{S}=\ddot{M} \cap_{D} \ddot{N}$ where $\ddot{M}$ is $\mathfrak{M}_{\mathrm{D}}$-open and $\ddot{N} \in \mathfrak{M}_{\mathrm{D}}-\mathrm{RC}(\mathrm{X})$,

2. a $\mathfrak{M}_{\mathrm{D}}-\mathrm{t}$ set if $\mathfrak{M}_{\mathrm{DX}}-\operatorname{int}\left(\mathfrak{M}_{\mathrm{DX}}-\operatorname{cl}(\ddot{S})\right)=\mathfrak{M}_{\mathrm{DX}}-\operatorname{int}(\ddot{S})$,

3. a $\mathfrak{M}_{\mathrm{D}}-\mathfrak{B}$ set if $\ddot{S}=\ddot{M} \cap_{D} \ddot{N}$ where $\ddot{M}$ is $\mathfrak{M}_{\mathrm{D}}$-open and $\ddot{N}$ is a $\mathfrak{M}_{\mathrm{D}}-\mathrm{t}$ set,

4. a $\mathfrak{M}_{\mathrm{D}}-\mathfrak{h}$ set if $\mathfrak{M}_{\mathrm{DX}}-\operatorname{int}\left(\mathfrak{M}_{\mathrm{DX}}-\operatorname{cl}\left(\mathfrak{M}_{\mathrm{DX}}-\operatorname{int}(\ddot{S})\right)=\mathfrak{M}_{\mathrm{DX}}-\operatorname{int}(\ddot{S})\right.$,

5. a $\mathfrak{M}_{\mathrm{D}}-\mathcal{C}$ set if $\ddot{S}=\ddot{M} \cap_{D} \ddot{N}$ where $\ddot{M}$ is $\mathfrak{M}_{\mathrm{D}}$-open and $\ddot{N}$ is a $\mathfrak{M}_{\mathrm{D}}-\mathfrak{h}$ set.

Theorem 4.3. If $\ddot{A}$ and $\ddot{B}$ are two a $\mathfrak{M}_{\mathrm{D}}-\mathrm{t}$ sets of a space $\mathrm{X}$ satisfying property $I_{D}$, then $\ddot{A} \cap_{D} \ddot{B}$ is a $\mathfrak{M}_{\mathrm{D}}-\mathrm{t}$ set in $\mathrm{X}$.

\section{Proof:}

Since $\ddot{A} \subseteq_{D} \mathfrak{M}_{\mathrm{DX}}-\operatorname{cl}(\ddot{A}), \mathfrak{M}_{\mathrm{DX}}-\operatorname{int}\left(\ddot{A} \cap_{D} \ddot{B}\right) \subseteq_{D} \mathfrak{M}_{\mathrm{DX}}-\operatorname{int}\left(\mathfrak{M}_{\mathrm{DX}}-\right.$

$\operatorname{cl}\left(\ddot{A} \cap_{D} \ddot{B}\right) \subseteq_{D} \mathfrak{M}_{\mathrm{DX}}-\operatorname{int}\left(\mathfrak{M}_{\mathrm{DX}}-\operatorname{cl}(\ddot{A}) \cap_{D} \mathfrak{M}_{\mathrm{DX}}-\operatorname{int}(\ddot{B})\right)$

$=\mathfrak{M}_{\mathrm{DX}}-\operatorname{int}\left(\mathfrak{M}_{\mathrm{DX}}-\operatorname{cl}(\ddot{A})\right) \cap_{D} \operatorname{int}\left(\mathfrak{M}_{\mathrm{DX}}-\operatorname{cl}(\ddot{B})\right)=\mathfrak{M}_{\mathrm{DX}}-\operatorname{int}(\ddot{A}) \cap_{D} \mathfrak{M}_{\mathrm{DX}}-$

$\operatorname{int}(\ddot{A})$ (since $\ddot{A}$ and $\ddot{B}$ are a $\mathfrak{M}_{\mathrm{D}}-\mathrm{t}$ sets $)=\mathfrak{M}_{\mathrm{DX}}-\operatorname{int}\left(\ddot{A} \cap_{D} \ddot{B}\right)$. Thus $\mathfrak{M}_{\mathrm{DX}}-$ $\operatorname{int}\left(\mathfrak{M}_{\mathrm{DX}}-\operatorname{cl}\left(\ddot{A} \cap_{D} \ddot{B}\right)\right)=\mathfrak{M}_{\mathrm{DX}}-\operatorname{int}\left(\ddot{A} \cap_{D} \ddot{B}\right)$ and $\ddot{A} \cap_{D} \ddot{B}$ is a $\mathfrak{M}_{\mathrm{D}}-\mathrm{t}$ set.

Theorem 4.4. If $\ddot{A}$ is a $\mathfrak{M}_{\mathrm{D}}-\mathrm{t}$ set of $\mathrm{X}$ and $\ddot{A} \subseteq_{D} \ddot{B} \subseteq_{D} \mathfrak{M}_{\mathrm{DX}}-\operatorname{cl}(\ddot{A})$ then $\ddot{B}$ is a $\mathfrak{M}_{\mathrm{D}}-\mathrm{t}$ set.

\section{Proof:}

We note that $\mathfrak{M}_{\mathrm{DX}}-\operatorname{cl}(\ddot{B}) \subseteq_{D} \mathfrak{M}_{\mathrm{DX}}-\operatorname{cl}(\ddot{A})$. So we have $\mathfrak{M}_{\mathrm{DX}}-\operatorname{int}(\ddot{B}) \subseteq_{D} \mathfrak{M}_{\mathrm{DX}}-$ $\operatorname{int}\left(\mathfrak{M}_{\mathrm{DX}}-\operatorname{cl}(\ddot{B})\right) \subseteq_{D} \mathfrak{M}_{\mathrm{DX}}-\operatorname{int}\left(\mathfrak{M}_{\mathrm{DX}}-\operatorname{cl}(\ddot{A})=\mathfrak{M}_{\mathrm{DX}}-\operatorname{int}(\ddot{A}) \subseteq_{D} \mathfrak{M}_{\mathrm{DX}}-\operatorname{int}(\ddot{B})\right.$. Thus $\mathfrak{M}_{\mathrm{DX}}-\operatorname{int}(\ddot{B})=\mathfrak{M}_{\mathrm{DX}}-\operatorname{int}\left(\mathfrak{M}_{\mathrm{DX}}-\operatorname{cl}(\ddot{B})\right)$ and therefore $\ddot{B}$ is a $\mathfrak{M}_{\mathrm{D}}-\mathrm{t}$ set.

Remark 4.5. The double union of two a $\mathfrak{M}_{D}-\mathfrak{h}$ sets need not be a $\mathfrak{M}_{D}-\mathfrak{h}$ set for example.

Let $X=\left\{z_{1}, z_{2}, z_{3}\right\}$ and

$\mathfrak{M}_{\mathrm{DX}}=\left\{\ddot{\emptyset}_{D}, \ddot{X}_{D},\left\langle\left\{\mathrm{z}_{1}\right\},\left\{\mathrm{z}_{2}, \mathrm{z}_{3}\right\}\right\rangle,\left\langle\left\{\mathrm{z}_{2}\right\},\left\{\mathrm{z}_{1}, \mathrm{z}_{3}\right\}\right\rangle,\left\langle\left\{\mathrm{z}_{1}, \mathrm{z}_{2}\right\},\left\{\mathrm{z}_{3}\right\}\right\rangle\right\}$.

Then $\left\langle\left\{\mathfrak{z}_{1}\right\},\left\{\mathfrak{z}_{2}, \mathfrak{z}_{3}\right\}\right\rangle$ and $\left\langle\left\{\mathfrak{z}_{2}\right\},\left\{\mathfrak{z}_{1}, \mathfrak{z}_{3}\right\}\right\rangle\{b\}$ are a $\mathfrak{M}_{\mathrm{D}}-\mathfrak{h}$ set but $\left\langle\left\{\mathfrak{z}_{1}, \mathfrak{z}_{2}\right\},\left\{\mathfrak{z}_{3}\right\}\right\rangle$ is not a $\mathfrak{M}_{\mathrm{D}}-\mathfrak{h}$ set.

Remark 4.6. If $\left(X, \mathfrak{M}_{\mathrm{DX}}\right)$ have property $I_{D}$. Then the double intersection of any two a $\mathfrak{M}_{\mathrm{D}}-\mathfrak{h}$ set is a $\mathfrak{M}_{\mathrm{D}}-\mathfrak{h}$ set.

\section{Comparison}


Some Properties of Double Minimal Space

In this section, we will explain the relationships between the concepts mentioned in Section 4.

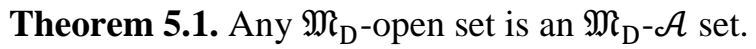

Proof: Direct from $\ddot{S}=\ddot{\mathrm{X}}_{D} \cap_{D} \ddot{S}$ where $\mathrm{X} \in \mathfrak{M}_{\mathrm{D}}-R C(\mathrm{X})$ and $\ddot{S}$ is $\mathfrak{M}_{\mathrm{D}}$-open.

Remark 5.2. The opposite of (Theorem 5.1) is n't true when all is said in done. Refer Example in (Remark 4.5), $\left\{\left\{\mathfrak{z}_{2}, \mathfrak{z}_{3}\right\},\left\{\mathfrak{z}_{1},\right\}\right\rangle$ is a $\mathfrak{M}_{\mathrm{D}}-\mathcal{A}$ set but not $\mathfrak{M}_{\mathrm{D}}$-open.

Theorem 5.3. Any $\mathfrak{M}_{\mathrm{D}}$-closed set is a $\mathfrak{M}_{\mathrm{D}}-\mathrm{t}$ set.

Proof: Let $\ddot{F}$ be $\mathfrak{M}_{\mathrm{D}}$-closed set, then $\ddot{F}=\mathfrak{M}_{\mathrm{DX}}-\operatorname{cl}(\ddot{F})$. So $\mathfrak{M}_{\mathrm{DX}}-\operatorname{int}(\ddot{F})=$ $\mathfrak{M}_{\mathrm{DX}}-\operatorname{int}\left(\mathfrak{M}_{\mathrm{DX}}-\operatorname{cl}(\ddot{F})\right)$. Hence $\ddot{F}$ is a $\mathfrak{M}_{\mathrm{D}}-\mathrm{t}$ set.

Remark 5.4. The opposite of (Theorem 5.3) isn't true when all is said in done. Refer Example in (Remark 4.5), $\left\langle\left\{\mathfrak{z}_{1}\right\},\left\{\mathfrak{z}_{2} \mathfrak{z}_{3}\right\}\right\rangle\{a\}$ is a $\mathfrak{M}_{\mathrm{D}}-\mathrm{t}$ set but not $\mathfrak{M}_{\mathrm{D}}$-closed.

Theorem 5.5. A Regular $\mathfrak{M}_{\mathrm{D}}$-open set is a $\mathfrak{M}_{\mathrm{D}}-\mathrm{t}$ set.

Proof: Let $\ddot{S}$ be Regular $\mathfrak{M}_{\mathrm{D}}$-open set, then $\ddot{A}=\mathfrak{M}_{\mathrm{DX}}-\operatorname{int}\left(\mathfrak{M}_{\mathrm{DX}}-\operatorname{cl}(\ddot{A})\right)$. So $\mathfrak{M}_{\mathrm{DX}}-\operatorname{int}(\ddot{A})=\mathfrak{M}_{\mathrm{DX}}-\operatorname{int}\left(\mathfrak{M}_{\mathrm{DX}}-\operatorname{cl}(\ddot{A})\right)$. Hence $\ddot{A}$ is a $\mathfrak{M}_{\mathrm{D}}-\mathrm{t}$ set.

Remark 5.6. The opposite of (Theorem 5.5) isn't true when all is said in done. Refer Example in (Remark 4.5), $\left\langle\left\{\mathfrak{z}_{3}\right\},\left\{\mathfrak{s}_{1}, \mathfrak{z}_{12}\right\}\right\rangle\{\mathrm{c}\}$ is a $\mathfrak{M}_{\mathrm{D}}-\mathrm{t}$ set but not Regular $\mathfrak{M}_{\mathrm{D}^{-}}$ open.

Theorem 5.7. Let $\left(X, \mathfrak{M}_{\mathrm{DX}}\right)$ have property $B_{D}$. Then every Regular $\mathfrak{M}_{\mathrm{D}^{-o p e n}}$ set is $\mathfrak{M}_{\mathrm{D}^{-} \text {-open. }}$

Proof: Suppose $\ddot{S}$ is Regular $\mathfrak{M}_{\mathrm{D}}$-open set, then $\ddot{S}=\mathfrak{M}_{\mathrm{DX}}-\operatorname{int}\left(\mathfrak{M}_{\mathrm{DX}}-\operatorname{cl}(\ddot{S})\right)$.

So $\mathfrak{M}_{\mathrm{DX}}-\operatorname{int}(\ddot{S})=\mathfrak{M}_{\mathrm{DX}}-\operatorname{int}\left(\mathfrak{M}_{\mathrm{DX}}-\operatorname{cl}(\ddot{S})\right)$ and we have $\ddot{S}=\mathfrak{M}_{\mathrm{DX}}-\operatorname{int}(\ddot{S})$.

By (Lemma 3.5), $\ddot{S}$ is $\mathfrak{M}_{\mathrm{D}^{-} \text {-open. }}$

Remark 5.8. The opposite of (Theorem 5.7) isn't true when all is said in done. Refer Example in (Remark 5.4), $\left\langle\left\{\mathfrak{z}_{1}, \mathfrak{z}_{2}\right\},\left\{\mathfrak{z}_{3},\right\}\right\rangle$ is $\mathfrak{M}_{\mathrm{D}}$-open but not Regular $\mathfrak{M}_{\mathrm{D}^{-}}$-open.

Theorem 5.9. Any a $\mathfrak{M}_{\mathrm{D}}-\mathrm{t}$ set is a $\mathfrak{M}_{\mathrm{D}}-\mathfrak{B}$ set.

Proof: Direct from $\ddot{S}=\ddot{X}_{D} \cap_{D} \ddot{S}$ where $\mathrm{X}$ is $\mathfrak{M}_{\mathrm{D}}$-open and $\ddot{S}$ is a $\mathfrak{M}_{\mathrm{D}}-\mathrm{t}$ set.

Remark 5.10. The opposite of (Theorem 5.9) isn't true when all is said in done, for example.

Let $X=\left\{\mathbf{z}_{1}, \mathbf{z}_{2}, \mathbf{z}_{3}\right\}$ and

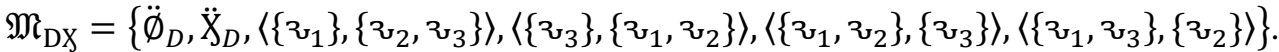

Then $\left\langle\left\{\mathfrak{z}_{1}\right\},\left\{\mathfrak{z}_{2}, \mathfrak{z}_{3}\right\}\right\rangle$ is a $\mathfrak{M}_{\mathrm{D}}-\mathfrak{B}$ set but not a $\mathfrak{M}_{\mathrm{D}}-\mathrm{t}$ set.

Theorem 5.11. Any $\mathfrak{M}_{\mathrm{D}}$-open set is a $\mathfrak{M}_{\mathrm{D}}-\mathfrak{B}$ set. 


\section{Dheargham Ali Abdulsada}

Proof: Since $\ddot{S}=\ddot{X}_{D} \cap_{D} \ddot{S}$ where $\ddot{S}$ is $\mathfrak{M}_{\mathrm{D}^{-o p e n}}$ and $\ddot{\mathrm{X}}_{D}$ is Regular $\mathfrak{M}_{\mathrm{D}^{-o p e n} \text {, by }}$ (Theorem 5.5), $\mathrm{X}$ is a $\mathfrak{M}_{\mathrm{D}}-\mathrm{t}$ set. By (Theorem 5.9) the proof is completed.

Remark 5.12. The opposite of (Theorem 5.11) isn't true when all is said in done. Refer Example in (Remark 5.4), $\left\langle\left\{\boldsymbol{z}_{3}\right\},\left\{\boldsymbol{z}_{1}, \boldsymbol{z}_{2}\right\}\right\rangle$ is a $\mathfrak{M}_{\mathrm{D}}-\mathfrak{B}$ set but not $\mathfrak{M}_{\mathrm{D}}$-open.

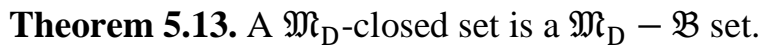

Proof: Obvious from (Theorem 5.3) and (Theorem 5.9).

Theorem 5.14. Assume $\left(X, \mathfrak{M}_{\mathrm{DX}}\right)$ have property $B_{D}$. Then every a $\mathfrak{M}_{\mathrm{D}}-\mathcal{A}$ set is a $\mathfrak{M}_{\mathrm{D}}-\mathfrak{B}$ set.

Proof: $\ddot{S}=\ddot{X}_{D} \cap_{D} \ddot{S}$ where $\ddot{X}_{D}$ is $\mathfrak{M}_{\mathrm{D}^{-o p e n}}$ and $\ddot{S}$ is Regular $m$-closed. Since $\ddot{S}$ is $\mathfrak{M}_{\mathrm{D}^{-}}$ closed, by (Theorem 5.3), $\ddot{S}$ is a $\mathfrak{M}_{\mathrm{D}}-\mathrm{t}$ set. By (Theorem 5.13), the proof is completed.

Remark 5.15. The opposite of (Theorem 5.14) isn't true when all is said in done. Refer Example in (Remark 5.4), $\left\langle\left\{\mathfrak{z}_{3}\right\},\left\{\mathfrak{z}_{1}, \mathfrak{z}_{2}\right\}\right\rangle\{\mathrm{c}\}$ is a $\mathfrak{M}_{\mathrm{D}}-\mathfrak{B}$ set but not a $\mathfrak{M}_{\mathrm{D}}-\mathcal{A}$ set.

Theorem 5.16. Any a $\mathfrak{M}_{D}-t$ set is a $\mathfrak{M}_{D}-\mathfrak{h}$ set.

Proof: Assume $\ddot{S}$ is a $\mathfrak{M}_{\mathrm{D}}-\mathrm{t}$ set, then $\mathfrak{M}_{\mathrm{DX}}-\operatorname{int}(\ddot{S})=\mathfrak{M}_{\mathrm{DX}}-\operatorname{int}\left(\mathfrak{M}_{\mathrm{DX}}-\operatorname{cl}(\ddot{S})\right)$, so $\mathfrak{M}_{\mathrm{DX}}-\operatorname{cl}\left(\mathfrak{M}_{\mathrm{DX}}-\operatorname{int}(\ddot{S})\right)=\mathfrak{M}_{\mathrm{DX}}-\operatorname{cl}\left(\mathfrak{M}_{\mathrm{DX}}-\operatorname{int}\left(\mathfrak{M}_{\mathrm{DX}}-\operatorname{cl}(\ddot{S})\right)\right)$, implies $\mathfrak{M}_{\mathrm{DX}}-$ $\operatorname{int}\left(\mathfrak{M}_{\mathrm{DX}}-\operatorname{cl}\left(\mathfrak{M}_{\mathrm{DX}}-\operatorname{int}(\ddot{S})\right)\right)=\mathfrak{M}_{\mathrm{DX}}-\operatorname{int}\left(\mathfrak{M}_{\mathrm{DX}}-\operatorname{cl}(\ddot{S})\right)$. The proof is completed.

Remark 5.17. The opposite of (Theorem 5.16) isn't true when all is said in done, for Example. Let $X=\left\{\mathbf{z}_{1}, \mathbf{z}_{2}, \mathbf{z}_{3}\right\}$ and

$\mathfrak{M}_{\mathrm{DX}}=\left\{\ddot{\emptyset}_{D}, \ddot{X}_{D},\left\langle\left\{\mathfrak{z}_{1}, \boldsymbol{z}_{2}\right\},\left\{\boldsymbol{z}_{3}\right\}\right\rangle,\left\langle\left\{\boldsymbol{z}_{2}, \boldsymbol{z}_{3}\right\},\left\{\boldsymbol{z}_{1}\right\}\right\rangle\right\}$. Then $\left\langle\left\{\mathfrak{z}_{2}\right\},\left\{\mathfrak{z}_{1}, \boldsymbol{z}_{3}\right\}\right\rangle$ is a $\mathfrak{M}_{\mathrm{D}}-\mathfrak{h}$ set but not a $\mathfrak{M}_{\mathrm{D}}-\mathrm{t}$ set.

Theorem 5.18. Any a $\mathfrak{M}_{\mathrm{D}}-\mathfrak{h}$ set is a $\mathfrak{M}_{\mathrm{D}}-\mathcal{C}$ set.

Proof: Obvious from $\ddot{S}=\ddot{X}_{D} \cap_{D} \ddot{S}$ where $\ddot{X}_{D}$ is $\mathfrak{M}_{\mathrm{D}}$-open and $\ddot{S}$ is a $\mathfrak{M}_{\mathrm{D}}-\mathfrak{h}$ set.

Remark 5.19. The opposite of (Theorem 5.18) isn't true when all is said in done. Refer Example in (Remark 5.10), $\left\langle\left\{\mathfrak{z}_{1}\right\},\left\{\mathfrak{z}_{2}, \mathfrak{z}_{3}\right\}\right\rangle$ is a $\mathfrak{M}_{\mathrm{D}}-\mathcal{C}$ set but not a $\mathfrak{M}_{\mathrm{D}}-\mathfrak{h}$ set.

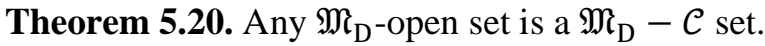

Proof: Obvious from $\ddot{S}=\ddot{X}_{D} \cap_{D} \ddot{S}$ where $\ddot{X}_{D}$ is a $\mathfrak{M}_{\mathrm{D}}-\mathfrak{h}$ set and $\ddot{S}$ is $\mathfrak{M}_{\mathrm{D}^{- \text {open }}}$

Remark 5.21. The opposite of (Theorem 5.20) isn't true when all is said in done. Refer Example in (Remark 5.4), $\left\langle\left\{\mathfrak{z}_{3}\right\},\left\{\mathfrak{z}_{1}, \mathbf{z}_{2}\right\}\right\rangle$ is a $\mathfrak{M}_{\mathrm{D}}-\mathcal{C}$ set but not $\mathfrak{M}_{\mathrm{D}^{-o p e n}}$.

Theorem 5.22. Any a $\mathfrak{M}_{\mathrm{D}}-\mathfrak{B}$ set is a $\mathfrak{M}_{\mathrm{D}}-\mathcal{C}$ set.

Proof: Since $\ddot{S}=\ddot{X}_{D} \cap_{D} \ddot{S}$ where $\ddot{X}_{D}$ is $\mathfrak{M}_{\mathrm{D}}$-open and $\ddot{S}$ is a $\mathfrak{M}_{\mathrm{D}}-\mathrm{t}$ set. By (Theorem 5.16), $\ddot{S}$ is a $\mathfrak{M}_{\mathrm{D}}-\mathfrak{h}$ set. The proof is complete from (Theorem 5.18). 
Some Properties of Double Minimal Space

Remark 5.23. The opposite of (Theorem 5.22) isn't true when all is said in done. Refer Example in (Remark 5.17), $\left\langle\left\{\mathfrak{z}_{2}\right\},\left\{\mathfrak{z}_{1}, \mathfrak{z}_{3}\right\}\right\rangle$ is a $\mathfrak{M}_{\mathrm{D}}-\mathcal{C}$ set but not a $\mathfrak{M}_{\mathrm{D}}-\mathfrak{B}$ set.

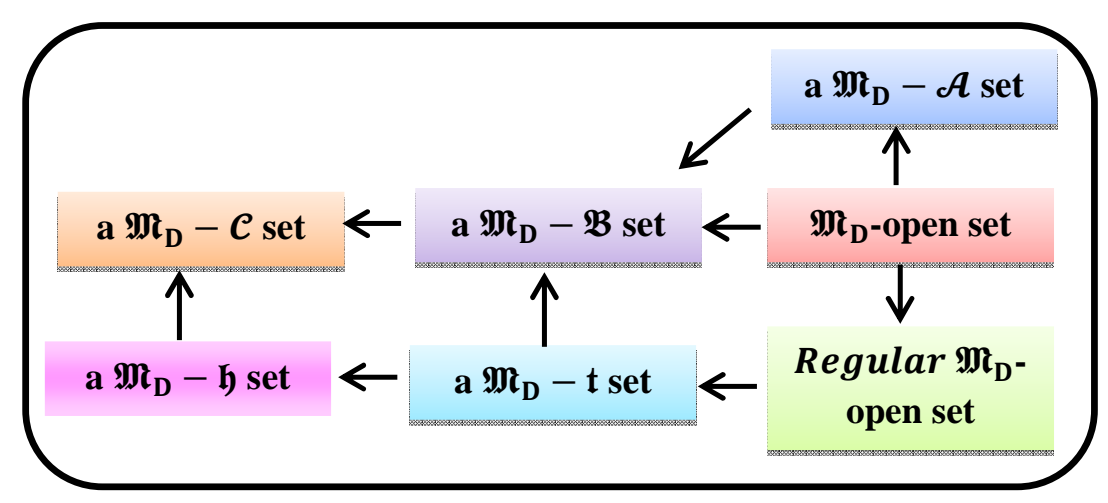

\section{REFERENCES}

1. A. Tarasov, Intuitionistic fuzzy sets, VII ITKR's Session,Sofia, Bulgarian (1983).

2. A.K.Adak, M.Bhowmik and M.Pal, Intuitionistic fuzzy block matrix and its some properties, Annals of Pure and Applied Mathematics, 1(1) (2012) 13-31.

3. D.Coker, A note on intuitionistic sets and intuitionistic points, Tr.J. Math, 20 (1996) 343-351.

4. D.Coker, An introduction to Intuitionistic topological spaces, Useful, 81 (2000) 5156.

5. E.Ahmed, M.S.Hossain and D.M.Ali, On intuitionistic fuzzy R0-spaces, Annals of Pure and Applied Mathematics, 10 (2015) 7-14.

6. J.G.Garcia and S.E.Rudabaugh,Order theoretic topological, categorical redundancies of interval valued sets, grey sets, vague sets, interval-valued doublesets, intuitionistic fuzzy sets and topologies, Fuzzy sets and Systems, 156(3) (2005) 445-484.

7. L.A.Zadeh, Fuzzy sets, Information and Control, 8 (1965) 338-353.

8. R.Islam, M.S.Hossain and M.R.Amin, Some remarks on intuitionistic $L-T_{2}$ spaces, Annals of Pure and Applied Mathematics, 13 (2) (2017) 249-255.

9. M.Bhowmik and M.Pal, Some results on intuitionistic fuzzy matrices and intuitionistic circulant fuzzy matrices, International Journal of Mathematical Sciences, 7 (1-2) (2008) 177-192.

10. M.Bhowmik and M.Pal, Some results on generalized interval-valued intuitionistic fuzzy sets, International Journal of Fuzzy Systems, 14 (2) (2012) 193-203. 